\title{
Estrategias para actuar en épocas de incertidumbre: una propuesta financiera
}

\author{
Alberto Parra Barrios
}

Recibido: 20 de agosto de 2020

Aprobado: 28 de septiembre de 2020

Parra, A. (2020). Estrategias para actuar en épocas de incertidumbre: una propuesta financiera. Revista Activos, 18(2), 165-204. https://doi. $\operatorname{org} / 10.15332 / 25005278 / 6265$

\section{Clasificación JEL: G01}

\section{Resumen}

Los ciclos económicos marcados por una depresión o recesión en las economías a nivel mundial llevan a los empresarios y directivos a implementar

\footnotetext{
* Administrador de empresas por la Universidad Javeriana, especialista en Finanzas por la Universidad de Los Andes, magíster en Finanzas y Mercados Financieros por la Universidad San Pablo CeU con énfasis en Finanzas por la Universidad Sergio Arboleda, en Economía por la Universidad Javeriana, y en Administración Financiera por la Universidad Sergio Arboleda. Docente de finanzas en el posgrado de la Universidad Sergio Arboleda y docente investigador en la Fundación Universitaria Cervantes San Agustín.

Correo electrónico: albertoparra9@yahoo.com

oRCID: http://orcid.org/0000-0003-1173-3073
} 
estrategias para garantizar el crecimiento y la supervivencia de sus empresas en un contexto de incertidumbre. Este documento pretende ofrecer una guía a los gerentes de finanzas, mostrando un marco que tenga en cuenta la estrategia y la aplicación de herramientas enfocadas a la obtención de liquidez, mediante la reactivación de los negocios por medio de la innovación y la creatividad, para evitar una quiebra empresarial. Los resultados destacan la necesidad de contar con estrategias empresariales adaptadas mediante la innovación, la creatividad, la participación del talento humano en la toma de decisiones y la construcción de un cuadro de gestión con indicadores, que le permitan al gerente de finanzas tener una radiografía permanente de su empresa y le den una alerta temprana.

Palabras clave: ciclos económicos, gerencia estratégica, flujo de caja.

\title{
Strategies for acting in times of uncertainty: a financial proposal
}

\begin{abstract}
Economic cycles marked by a depression or recession in economies worldwide, lead entrepreneurs and managers to implement strategies to ensure the growth and survival of their companies, in a context of uncertainty. The purpose of this document is to offer a guideline to financial managers, showing a framework that takes into account the strategy and the application of tools focused on obtaining liquidity, through the reactivation of business through innovation and creativity, to avoid business bankruptcy. The conclusions highlight the need to have business strategies adapted through innovation and creativity, the participation of human talent in decision-making and the construction of a management chart with indicators that allow the financial manager to have a permanent X-ray of his company, with indicators that give an early warning.
\end{abstract}

Keywords: economic cycles, strategic management, cash flow. 


\section{Introducción}

Antes de que apareciera la pandemia por la covid-19, los organismos internacionales como el Banco Mundial y el Fondo Monetario Internacional preveían que el crecimiento económico mundial en el 2020 estaría en el 2.5 \% ("Perspectivas económicas mundiales", 2020). Para el mes de junio, en el comunicado de prensa del Banco Mundial ("La covid-19 hunde a la economía mundial”, 2020) se consignó que la economía se reduciría en un $5.2 \%$ durante el 2020 y que habría un repunte del $4.2 \%$ en 2021, aunque con perspectivas inciertas, esperando que la situación empeore.

Curiosamente, al aparecer la abrupta desaceleración del Producto Interno Bruto (РIB) en febrero de 2020, los consultores y asesores financieros iniciaron una serie de recomendaciones para actuar ante la crisis, etiquetando la gestión a realizar en las empresas con la frase "cómo actuar en épocas de coronavirus". Ahora bien, los ciclos económicos siempre han estado presentes y en las etapas de depresión y recesión es cuando aparecen las crisis. Estos ciclos tienen diferente duración por lo que, en muchos casos, salir de una crisis puede llevar su tiempo.

Desde comienzos del siglo xxI, las transformaciones digital y empresarial ya experimentaban un cambio importante; no en vano se ha denominado a esta época "la era de las comunicaciones", debido a los grandes avances que, desde finales del siglo xx, la tecnología, la informática y la digitalización han presentado. Las herramientas financieras para ayudar a los gerentes a alcanzar sus propósitos económicos se han desarrollado desde la década de 1950, con énfasis en los años ochenta, gracias a las aportaciones teóricas sobre la economía financiera de la empresa, la eficiente gestión de tesorería y la generación de valor para los accionistas.

A partir del 2010, las organizaciones comenzaron a implementar plataformas colaborativas robustas e inversión en infraestructura tecnológica, y a utilizar instrumentos muy eficientes para posicionarse en el mercado, como la investigación del consumidor a través de la inteligencia de mercados y la inteligencia de negocios para ofrecer nuevos productos. Sumado 
a esto, por muchos años se ha trabajado en la planeación a largo plazo y la implementación de cursos de acción para lograr los objetivos económicos y financieros. Para ello, los gerentes utilizan en sus empresas la "gerencia estratégica" en la cual fijan sus actuaciones.

Dada la existencia de herramientas suficientes, surge la siguiente pregunta: ¿sin excepción alguna, los gerentes generales y los gerentes de finanzas estaban conscientes de la importancia de estas herramientas y estaban preparados para afrontar una situación inesperada? Sin temor a equívocos, se puede decir que ellos han olvidado los principios financieros y la necesidad de innovar constantemente en estrategias empresariales, porque sus empresas han mostrado un resultado financiero exitoso y han vivido en un ambiente de prosperidad empresarial. Sin embargo, hay que recordar que aquellas empresas que aplican regularmente los principios de gerencia estratégica y los principios financieros son las que pueden afrontar con éxito los problemas causados por las crisis.

El presente trabajo pretende ayudar al gerente financiero a identificar los problemas que impactan el área financiera como consecuencia de las crisis económicas; además, entrega unos instrumentos teóricos y prácticos que le permitan enfrentar los choques a los que se vea expuesto. Entre los instrumentos sobre los que se puede apoyar para su gestión se encuentran el flujo de caja; las estrategias para su elaboración; la medición del riesgo de liquidez; el modelo económico y los submodelos que lo conforman; el punto de equilibrio y la utilización de nuevos instrumentos que fortalecen la gestión como el Desing Thinking.

Para alcanzar este propósito, se parte de la fundamentación teórica y los instrumentos presentados por economistas y financieros en los últimos años, quienes reafirman los principios, las estrategias y las técnicas para realizar una gestión financiera con éxito, así como para la creación de nueva riqueza para el accionista, enfrentando la incertidumbre ante choques externos inesperados. En ese contexto, se enfatiza el campo de acción del gerente financiero con un enfoque administrativo; además, se diseña un 
plan para enfrentar la crisis aplicando los principios y las herramientas mencionadas.

La metodología empleada en el documento se basa en el estudio exploratorio de autores que han investigado los componentes más relevantes del tema económico financiero y de estrategia empresarial, buscando la correlación entre ambos. Esta exploración tiene en cuenta que aquellos autores también aborden la utilización de otras herramientas actuales como la inteligencia digital y big data que hoy ayudan a la monetización de los planes, programas financieros y de mercados, además de algunos aportes de estrategia empresarial.

En la revisión de la literatura se destacan los principales economistas que han contribuido al fortalecimiento de la gerencia estratégica empresarial y la gerencia financiera en los últimos años. Se parte de las orientaciones de Ezra Solomon (1972) quien fija el alcance de las decisiones financieras en la empresa, la necesidad de administrar los riesgos en las organizaciones y, en este caso, el riesgo de liquidez (LAR, por sus siglas en inglés), que tiene alta importancia en situaciones de incertidumbre.

Finalmente, se presentan unas recomendaciones para que el gerente de finanzas tenga en cuenta en su gestión diaria, y unos fundamentos e instrumentos básicos para enfrentar lo mejor posible los impactos externos en su empresa. Entre estos elementos, se destaca, por una parte, la gestión hacia el flujo de tesorería, que es la herramienta más importante en épocas de incertidumbre puesto que muestra la liquidez de la empresa, y por tanto, la necesidad de medir el riesgo de liquidez. Por otra parte, se remarca la implementación de la gerencia estratégica en la empresa y el cuadro de indicadores de gestión financiera que permiten determinar con alerta temprana las posibles dificultades financieras, para actuar anticipadamente y hacer partícipes a los colaboradores en la toma de decisiones a todo nivel y utilizar herramientas de inteligencia digital. Asimismo, se presentan anexos, a manera de cuadros ilustrativos, para recordar a los analistas financieros los modelos e instrumentos que ayudan a identificar los problemas y obtener indicadores financieros de gestión. 


\section{Marco teórico}

Los avances en materia financiera, en estrategia empresarial y en tecnología para las comunicaciones han sido importantes en los últimos años y de gran utilidad para la toma de decisiones de los gerentes de finanzas, quienes tienen la responsabilidad de generar valor a los grupos de interés vinculados a las empresas.

La revisión de la literatura realizada en este trabajo parte, en primer lugar, de las precisiones de Ezra Solomon (1972) quien destaca el alcance de la función financiera en las empresas visto como una parte del fenómeno administrativo, en lugar de limitarse a la obtención de fondos. Entonces, aborda el tema de la producción, la comercialización, la administración propiamente dicha y otras divisiones donde se toman decisiones con respecto a la adquisición de activos y movimientos de fondos que comprometen la eficiencia en las operaciones financieras.

En segundo lugar, se aborda el trabajo de Copeland et al. (2004) quienes recaban en la necesidad de concentrarse en los rendimientos del flujo de caja a largo plazo, resaltando la importancia de la liquidez en la empresa mientras se busca la rentabilidad de las inversiones, tomando conciencia de la generación de valor por medio de la cultura organizacional y la gestión del rendimiento en cada una de las unidades del negocio.

Posteriormente, Zvi Bodie y Robert Merton (1999) precisan que las decisiones en una empresa son, al menos parcialmente, de tipo financiero, porque requieren de un compromiso entre costos y beneficios a largo plazo que involucra la planeación estratégica. Además, aseguran que la responsabilidad del director financiero incluye administrar el capital de trabajo, administrar la exposición a los riesgos, el uso de la tecnología y el análisis de preferencias al consumidor para predecir los cambios futuros para el logro de los objetivos económicos empresariales.

Por su parte, Block y Hirt (2001) presentan un análisis sobre la gerencia del capital de trabajo y las decisiones financieras frente a la liquidez. Para 
ello, analizan el crecimiento de los activos corrientes, la pronosticación de las ventas y el equilibrio con los programas de producción. Además, estudian las alternativas de financiación a corto y a largo plazo y recalcan que se debe contar con una planeación financiera adecuada y una política óptima en los componentes del capital de trabajo.

Con relación al trabajo de la gerencia estratégica, orientada a la creación de valor, Fred David (1991) entrega las técnicas de gerencia estratégica empresarial y presenta un formato para realizar el diagnóstico interno finalizando con la matriz de evaluación. Mientras que, Mintzberg y Brian (1997) desarrollan los procesos a través de los cuales se establece la estrategia, analizan la cadena de valor donde incluyen la tecnología, la administración del recurso humano e infraestructura, la diferenciación del producto, el desarrollo de una competencia distintiva y las actividades primarias, para la comercialización y ventas.

Los autores anteriores coinciden en muchos aspectos con Kaplan y Norton (2005) quienes trabajan la construcción del mapa estratégico para crear valor, a partir de cuatro perspectivas ya conocidas ampliamente. Se destaca la perspectiva de aprendizaje y crecimiento porque permiten identificar los activos intangibles, como el capital de la información, el capital humano y el capital organizacional como elementos fundamentales para obtener la creación de valor por medio de mejores ingresos y el crecimiento empresarial.

Por último, al tomar relevancia la innovación, W. Chan Kim y Mauborgne (2015) actualizan los conceptos de estrategia al centrar la gestión en la creación de nuevos mercados en áreas que no están explotadas y que generan oportunidades de crecimiento rentable y sostenido a largo plazo. Esto se da en la innovación orientada a la creación de valor para el consumidor, que permita generar caja a largo plazo aplicando principios de formulación y ejecución que atenúen el riesgo. 


\section{Plan de trabajo para enfrentar las crisis}

El plan de trabajo para enfrentar los cambios que se generan por los choques económicos externos está relacionado con la planeación estrategia de la empresa. Se inicia con la auditoría completa de la empresa, aplicando las pautas que recomiendan autores mencionados, como se observa a continuación.

\section{Análisis estratégico}

La mejor defensa en épocas de incertidumbre es contar con un plan estratégico que neutralice o minimice los efectos negativos de los períodos de crisis. Estar preparado es la mejor estrategia, una defensa es vital para actuar en momentos de choques externos.

Antes de abordar el análisis de variables, es importante aclarar cuál es el negocio de la empresa. Para resolver este interrogante hay que responder a las preguntas: ¿cuál es realmente mi negocio?, ¿qué es lo que soluciona mi negocio?, ¿quién es mi cliente?, y ¿porqué me compra?

Una visión abierta del negocio permite ampliar productos y servicios para crecer. Esto es importante porque ante la crisis, hay que ser creativos e innovar, no temer al cambio: las crisis sirven para mejorar y para crecer.

\section{Análisis externo antes de la crisis}

Las finanzas no están separadas de la economía, por el contrario, las finanzas empresariales forman parte de la economía financiera de la empresa, y esta a su vez se integra dentro de un cuerpo superior que es la economía. La historia nos muestra que los nuevos aportes al conocimiento financiero provienen de investigaciones económicas, del uso de la matemática, la estadística y la psicología.

Entonces, es muy valioso estar informado sobre cómo se encuentra la economía y cuáles son sus tendencias en cuanto a PIB, inflación, tasas de 
interés, tipo de cambio, productividad de la mano de obra, déficit de cuenta corriente, balanza de capitales, déficit fiscal, inversión extranjera directa, reservas internacionales, desempleo. Además, dado que la empresa trabaja en un sector específico, es importante saber cómo se encuentra el sector en el que opera la empresa, considerando los factores que la hacen vulnerable (Parra, 2014). Para mantenerse informado, solo basta con ingresar a la página del Banco Central, revisar las decisiones sobre política monetaria que tomen los miembros de la junta directiva del Banco de la República o el Sistema de Reserva Federal en Estados Unidos y observar las publicaciones de revistas especializadas en el tema.

Simultáneamente, es preciso informarse sobre temas externos relacionados con el mercado que se comparte, las tendencias del consumidor, los precios, identificar y evaluar las características de los competidores, las fuerzas y tendencias tecnológicas y la regulación desde el contexto empresarial en el que se encuentra la empresa.

\section{Análisis interno antes de la crisis}

En esta etapa se busca conocer cómo estaba operando la empresa antes de presentarse la crisis. Para lograr ese fin, se formulan las preguntas: ¿se cumplía con los indicadores establecidos?, ¿cómo estaban las relaciones internas entre áreas funcionales?, ¿existía una matriz de evaluación de factores?, ¿cómo era la posición financiera, comercial, de mercadeo y de relaciones humanas? Para responder a estos cuestionamientos, David (1991) propone un modelo de auditoría integral cuyos principales elementos de análisis son

- Auditoría gerencial, enfocada a la gerencia estratégica.

- Auditoria comercial y de mercadeo, orientada a un proceso de creación de valor con productos de buena calidad ${ }^{1}$.

1 ¿Cuál era el nivel de satisfacción de los clientes?, ¿con qué periodicidad se hacían las compras?, ¿cuál era el comportamiento de las ventas?, ¿cómo se planificaban los 
- Auditoría de producción, para obtener un proceso de producción que optimice los costos y la productividad.

- Auditoria financiera, con el fin de determinar la situación económica y financiera de la empresa por medio de indicadores de liquidez, endeudamiento, rentabilidad y eficiencia. Así, analizar si la gerencia de finanzas logra una buena gestión por medio de la eficiencia en la aplicación de las funciones financieras básicas ${ }^{2}$. En esta área se aplican los siguientes pasos:

$\triangleright$ Diagnóstico financiero: su fin es diagnosticar la gestión y la política financiera de la empresa. Se mide a través de cuadros de indicadores que muestren el desarrollo histórico para un período de cinco años. Este diagnóstico se basa en el cumplimiento de los objetivos establecidos en la planeación financiera, para tomar los correctivos anticipándose a los eventos futuros. Entonces, se evalúa si la empresa está en capacidad de obtener capital a corto y a largo plazo; además, se revisa si existe una estructura consistente para hacer planeación financiera, elaborar presupuestos y flujo de caja.

$\triangleright$ Determinar cuál fue el impacto de la crisis en cada una de las dependencias de la empresa y las áreas a su cargo; esto se realiza por medio de los indicadores.

$\triangleright$ Cuadro de indicadores de gestión financiera: para poder actuar oportunamente, el gerente de finanzas debe contar con un tablero de control actualizado que le muestre cómo se encuentra financieramente la empresa y le permita hacer análisis de sensibilidad, tomar decisiones a partir de cambios en las

servicios y productos?, ¿cómo se establecía el precio?, ¿la distribución del producto era adecuada y oportuna?, ¿qué resultados mostraba la investigación de mercados?

2 Las decisiones de inversión en activos productivos, decisiones de financiamiento y de liquidez y pago de dividendos, se conocen como las tres funciones principales del gerente de finanzas. 
políticas de capital de trabajo, la estructura de endeudamiento, el comportamiento de las ventas, el control de costos y gastos, y la generación de valor. Estos indicadores deben ayudarle a identificar cuál es la ruta que debe seguir (Parra, 2014).

$\triangleright$ Estructura de costos: permite elaborar el Estado de resultados por contribución, explicado por Saldivar (p. 38) y desarrollado en el Anexo 1 y los diferentes puntos de equilibrio que permitan tomar decisiones financieras oportunas explicados en el Anexo 3.

$\triangleright$ Planeación financiera y presupuestos: permite saber si en el momento del choque económico, la empresa está cumpliendo con los presupuestos establecidos para cada una de las dependencias y conocer el nivel de liquidez en el flujo de tesorería.

Estos instrumentos muestran cómo se encuentra la empresa antes de la crisis respondiendo a las preguntas:

- ¿Se cumplen los indicadores de liquidez, capital de trabajo, endeudamiento y rentabilidad?

- ¿Hay generación de valor económico agregado (EvA)?

- ¿Se está generando caja para cumplir con las obligaciones financieras y económicas?

- ¿Se presentan dificultades para el punto de equilibrio?

- ¿Qué desviaciones hay en los presupuestos establecidos?

- ¿La estructura de costos permite ejercer un control sobre la productividad, el margen comercial, elaborar el estado de resultados por contribución real de la empresa? 
Con los resultados anteriores se construye una matriz de evaluación o de impacto que muestre la radiografía de la empresa a la fecha del choque externo, incluyendo indicadores financieros básicos (David, 1991).

\section{Análisis externo después de la crisis}

Comprende la identificación y evaluación de tendencias, hechos políticos, sociales, económicos, tecnológicos y competitivos que son claves cuando se presenta la crisis.

Después de establecer cuál es la situación, el gerente de finanzas debe responder a la pregunta: ¿cómo afecta a la empresa esta situación económica mundial y nacional de cara al futuro? El resultado de la evaluación de la situación a partir de la crisis se da a partir de un conjunto de variables e indicadores críticos sobre los cuales debe construirse el futuro de la empresa, determinando qué oportunidades que se presentan y qué amenazas debe tratar de superar. Los objetivos de las estrategias empresariales formulas son el aprovechamiento de las oportunidades y la reducción del impacto de las amenazas.

Entonces, gracias al análisis de la situación externa a partir del momento en que se acentúa la crisis económica, es posible observar:

- Estado y tendencias de la economía global y de la economía nacional: se evidencia el crecimiento del producto interno bruto potencial; el crecimiento del sector económico de la empresa; las variables económicas estimadas sobre desempleo, inflación y tasa de cambio representativa del mercado (TRM); los índices accionarios; la política salarial; las medidas económicas y monetarias adoptadas por el Gobierno para rescatar la economía; las ayudas monetarias, el apoyo y las ayudas laborales; $y$, las opciones para financiar y refinanciar los sectores económicos. Además, se incluye el análisis de la tendencia del mercado que comparte la empresa y se observa si hay posibilidad de que perder o ganar participación. 
- Las tendencias sociales por área geográfica: nivel de educación, actitudes de compra y de crédito, y cambios en la población.

- Las tendencias políticas a nivel internacional: especialmente en los países con los que se mantienen relaciones comerciales, sus nuevas regulaciones, aranceles y poder de grupos de interés.

- Las fortalezas de los competidores, sus debilidades y sus estrategias.

\section{Análisis interno después del impacto económico}

Las finanzas empresariales no trabajan en una campana de cristal, están vinculadas al comportamiento de todas las áreas y sus decisiones, en última instancia, afectan el flujo de caja, del cual el gerente de finanzas debe estar pendiente, dado que la empresa se quiebra por la iliquidez.

De acuerdo con la interpretación de Ezra Solomon cuando afirma que el enfoque de finanzas en las organizaciones es administrativo; en épocas de crisis e incertidumbre, para el gerente de finanzas es fundamental el analizar (con las áreas funcionales) cómo impacta la crisis a cada una de las dependencias. Aquel no debe olvidar que la creación de valor para el accionista está vinculada con la creación de valor para los stakeholders o grupos de interés, donde él tiene una responsabilidad compartida.

El gerente de finanzas puede emprender acciones como

- Establecer, con la gerencia general y las divisiones, los objetivos a cumplir a partir de los ajustes por la crisis.

- Determinar las nuevas oportunidades, fortalezas, debilidades y amenazas para el negocio.

- Analizar si es adecuada la estructura organizacional para afrontar la crisis, es decir, abordar los elementos siguientes: 
$\triangleright$ Qué estructura orgánica se necesita para llegar al cliente generándole valor.

$\triangleright$ Estudiar si es óptimo el estado de ánimo de los empleados para afrontar la crisis; hay que prepararlos para un cambio exitoso.

$\triangleright$ Establecer nuevos mecanismos de control y recompensa para los empleados.

$\triangleright$ Implementar en la empresa la cultura de la innovación y el análisis y raciocinio para resolver problemas (Design Thinking) para orientar las actividades a la creación de valor al cliente, mejorar los procesos con un enfoque participativo.

- Trabajar con el área de mercadeo:

$\triangleright$ En la implementación del método de inteligencia digital: para conocer el comportamiento del consumidor, crear y posicionarse en nuevos negocios y mercados, mejorar el flujo futuro de caja y mejorar la rentabilidad económica y financiera haciendo inteligencia de mercados e inteligencia de negocios, con el fin de encontrar nuevos mercados más allá de la demanda existente, desarrollar nuevos espacios de mercado, no disputados, donde la competencia sea irrelevante (W. Chan Kim, 2015).

$\triangleright$ Evaluar si la empresa ha reducido su participación en el mercado, cómo está la calidad de los productos, el servicio a los clientes y si los precios actuales son apropiados o deben fijarse nuevos precios.

$\triangleright$ Cómo se pueden diversificar los productos o servicios que se ofrecen en la actualidad para generar valor al consumidor.

$\triangleright$ Analizar si se pueden crear nuevas líneas de productos: ¿cómo puedo replantear mi negocio con lo que sé hacer?, ¿cómo se 
pueden diversificar los productos o servicios que ofrezco en la actualidad, para generar valor al consumidor?

$\triangleright$ Evaluar la efectividad de la actual estructura de ventas; si la actual estructura de ventas no es efectiva, es posible reducirla.

$\triangleright$ Evaluar desde su efectividad la actual estrategia de promoción y publicidad.

$\triangleright$ Evaluar cuánto cuesta la planificación de la función de mercadeo propuesta, puesto que esta afecta el presupuesto y el flujo de caja.

$\triangleright$ Si la empresa realiza operaciones de comercio exterior: analizar el mercado externo donde se tengan operaciones; estudiar la posibilidad de abrir nuevos mercados, considerando que esto es un proyecto de inversión a mediano plazo; identificar y diseñar una estrategia adecuada para aprovechar los incentivos del Gobierno y el posicionamiento externo; asegurar el pago de los clientes externos; y ejecutar nuevas formas de negociación.

$\triangleright$ Identificar la capacidad de producción con base en la demanda interna y externa, la capacidad de almacenamiento, los costos de producción y de exportación, los recursos financieros y los riesgos de exportar o importar.

- Desde el área de finanzas, es necesario trabajar en aspectos propios de la dependencia como:

$\triangleright$ Informarse a diario sobre los anuncios del Gobierno y las expectativas a futuro en materia de economía, finanzas y política monetaria.

$\triangleright$ Determinar la política financiera y los nuevos indicadores de acuerdo con impacto que recibió la empresa. 
$\triangleright$ Recuperar las cuentas por cobrar comerciales, el periodo de pago a proveedores y el período de rotación de inventarios. Para ello, es preciso plantear las preguntas: ¿cuál es el nuevo nivel de inventario necesario?, y ¿cuál es el nuevo costo por almacenamiento y por pedido?

$\triangleright$ Estimar el nuevo ciclo de comercialización y caja.

$\triangleright$ Definir el nivel de endeudamiento que se necesita para atender las nuevas necesidades de capital de trabajo; así como la garantía que se puede ofrecer a los acreedores.

$\triangleright$ Elaborar los nuevos presupuestos y flujo de caja.

$\triangleright$ Determinar cómo está el actual flujo de caja:

- ¿Se cuenta con los recursos necesarios para cancelar las obligaciones frente a los empleados?

- ¿Hay un flujo de caja para atender las obligaciones con proveedores?

- ¿Cómo se proyecta el cumplimiento de las actuales obligaciones frente a los proveedores y demás acreedores?

$\triangleright$ Establecer el estado de la empresa respecto al pago de las obligaciones financieras.

$\triangleright$ A partir del comportamiento del tipo de cambio, analizar el uso de futuros, opciones y compromisos de comprar o vender un activo a un precio fijado en una fecha determinada (forwards) e idear una estrategia de protección ante las posibles fluctuaciones en la divisa. En este punto, es importante evaluar el costo financiero para las obligaciones con bancos en el exterior. 
$\triangleright$ Registrar el saldo en tesorería y prever si hay dificultades de liquidez por un tiempo prolongado.

- Construir un modelo para medir el riesgo de liquidez 3 .

- Determinar la nueva estructura de costos de la empresa para conocer el nivel de costos fijos y variables con el objetivo de sensibilizar el rendimiento operativo.

- Optimizar los recursos observando el efecto de las decisiones en el índice Dupont (Sallenave, 1985), ver Anexo 4.

- Cuantificar, con el área de producción, el impacto de la crisis en los procesos de producción y el costo de los productos fabricados. Para esto, es recomendable:

$\triangleright$ Evaluar el impacto de la crisis con los proveedores de materia prima y las entregas de la materia prima.

$\triangleright$ Reconocer si la maquinaria y los equipos de producción se encuentran en buen estado, la capacidad instalada es suficiente para afrontar la crisis, y determinar la capacidad ociosa.

$\triangleright$ Detallar los procedimientos y políticas de control de calidad, inventarios y productividad del personal.

- Trabajar con el área de talento humano en el desarrollo organizacional de la empresa:

$\triangleright$ El desarrollo de la capacidad de los empleados mejora los procesos de optimización de las operaciones de la empresa. Este desarrollo se alcanza por medio de la motivación del capital

3 Por ejemplo, el modelo de riesgo de liquidez utilizando la volatilidad dinámica o con suavizamiento exponencial (Haro, 2004, p. 46). 
humano, la participación en la toma de decisiones ${ }^{4}$; con mayor razón, a partir de la crisis, el personal propone soluciones para el problema.

$\triangleright$ Es imprescindible la implementación de políticas para generar valor al personal de la empresa.

\section{Gestión estratégica desde la gerencia financiera}

Una vez el gerente de finanzas ha evaluado el impacto de las variables externas en las áreas funcionales de la empresa, aquel debe aplicar un enfoque estratégico. La mejor estrategia es estar preparado para el cambio, por lo tanto, es preciso implementar la gerencia estratégica financiera en la empresa.

En ese contexto, el gerente de finanzas seguramente tiene que replantear los objetivos y redefinirlos a corto, mediano y largo plazo. Con base en estos, debe fijar las nuevas políticas financieras en materia de liquidez y capital de trabajo, endeudamiento y distribución de utilidades. Debido a eso, es importante contar con las siguientes herramientas de gestión financiera:

- Una base correcta y sistemática que oriente los fondos hacia y en la empresa, para lograr los objetivos en materia financiera después de la crisis (Solomon, 1972). Esto incluye, en primer lugar, el presupuesto anual ajustado que orienta la canalización de los recursos a los diferentes departamentos de la empresa. Este presupuesto facilita la elaboración del flujo de caja actualizado, con base en las nuevas políticas y necesidades establecidas. Para esta labor, el gerente debe:

$\triangleright$ Cuantificar los gastos de cada una de las dependencias, con base en la nueva estructura orgánica y sus programas.

$4 \quad$ Kaplan y Norton (2005) sugieren posibles estrategias (p. 323). 
$\triangleright$ Construir el futuro de la empresa implementando instrumentos de planeación y análisis con base en el modelaje financiero.

$\triangleright$ Realizar periódicamente el análisis comparativo del presupuesto.

Asimismo, es necesario un control del nuevo flujo de caja, este permite hacer análisis de sensibilidad para conocer el impacto probable en cuentas de ingresos y egresos y actuar con anticipación. En ese orden de ideas, el gerente debe hacer análisis periódicos del ciclo de producción y ciclo de caja y analizar el efecto en el costo financiero y el rendimiento. Además, debe conversar con los proveedores y deudores, para conocer su situación y posición comercial y financiera.

- Un modelo de gerencia estratégica financiera $a^{5}$ a partir del estado de resultados por contribución: este enfoque se encuentra explicado en Horngren (1994, p. 141) y en Saldivar, (1999, p. 38), construido a partir de los costos variables, y sirve para tomar decisiones financieras, como se refleja en el Anexo 1. La implementación de ese modelo implica:

$\triangleright$ La construcción de un submodelo de estructura y un submodelo de contribución por productos ${ }^{6}$ que facilitan el análisis de sensibilidad en las cuentas (Anexo 1). Además, los elementos principales que deben determinarse en este proceso son, por un lado, los costos variables unitarios por producto, el margen de contribución unitario por producto y los costos fijos por producto; por otro lado, el punto de equilibrio por productos; $y$,

5 Este es un análisis de las estrategias generales para mejorar la utilidad de operación, a partir de la construcción de un estado de resultados con base en costos fijos y variables (estado de resultados por contribución) que se encuentra en Saldivar (1999, pp.37-69), también en Sallenave (1985, pp. 78-87).

6 El submodelo de estructura es la parte del estado de resultados del modelo económico de Saldivar (1999, p. 38) que se ubica en los costos fijos (entre el margen de contribución y el margen operacional); y el submodelo de contribución por productos es la parte que describe los costos variables por productos (entre los ingresos por ventas y el margen de contribución) descrito en Saldivar (1999, pp. 53 y 62). 
finalmente, el punto de equilibrio con descuento en el precio de venta.

$\triangleright$ Un nuevo punto de equilibrio para toma de decisiones. En el Anexo 3 se ilustra la formulación de diferentes puntos de equilibrio con base en el modelo planteado por Antonio Saldivar (1999).

$\triangleright$ El aumento del margen de contribución mediante el crecimiento de las ventas en unidades o la reducción de los costos variables.

$\triangleright$ La reducción del índice de estructura con base en el análisis de costos fijos. Para este propósito, el gerente debe analizar si es posible reducir los costos fijos de la empresa; eliminar los costos fijos provenientes de activos improductivos; definir cómo convertir los costos fijos en costos variables; ejemplo, un nuevo modelo de contratación de personal por prestación de servicios a tiempo definido o por vinculación de servicio entrega de proyecto; reducir costos fijos de áreas con índice de estructura alto; modificar los procesos para reducir costos y llegar con mayor eficiencia al consumidor final; implementar la Cadena de Abastecimiento Lean; y mejorar las áreas funcionales y la productividad.

- Una evaluación de la inversión en infraestructura tecnológica y metodologías de desarrollo: la tecnología juega un papel muy importante en la mejora de las operaciones y la solución de problemas en la cadena de abastecimiento. Además, permite una rápida retroalimentación con el consumidor y con los empleados, facilita el manejo de grandes volúmenes de datos e información, inicialmente puede incrementar costos, pero mejora los procesos y permite crecer a futuro los ingresos. En este aspecto, es necesario que, después de la crisis, el gerente analice cómo reducir los costos variables a partir de estrategias. 
- Indicadores financieros integrados: estos permiten tomar decisiones anticipadas para mejorar la rentabilidad. Para su construcción, el gerente sigue varios pasos: primero, determina el índice ROI o ROA (Anexo 4), el cual facilita las simulaciones de gestión financiera, operando los indicadores financieros que lo conforman. Segundo, determina el eva (Anexos 6 y 7) y hace simulaciones de gestión financiera para incrementar su valor, operando los componentes del indicador. Por último, reduce el costo de capital de la empresa.

- Un flujo de caja ajustado y basado en el modelo de flujo de caja más cercano al flujo de tesorería: este permite conocer los ingresos y egresos en el momento en que realmente ocurren y determinar la liquidez de la empresa, cuantificar las disponibilidades para optimizar los recursos en efectivo, mejorar las utilidades, identificar las necesidades de financiamiento y medir el riesgo de liquidez.

Ehrhardt y Brigham (2006) ofrece una guía para la construcción del nuevo flujo de caja (Anexo 2), cuando trata la administración del capital de trabajo y la construcción del flujo de efectivo, incluyendo una técnica para establecer nuevos indicadores en las cuentas que conforman el activo corriente y el pasivo corriente operativo.

Es importante que el gerente

$\triangleright$ inicie con la innovación puesto que la crisis cambia totalmente el panorama. De las crisis se puede salir rápidamente, con éxito, si se hacen las cosas lo mejor posible, se aprovechan las oportunidades y se innova;

$\triangleright$ prevea el futuro de la empresa a partir de los desembolsos de efectivo, teniendo en cuenta que se necesita trabajar con eficiencia y austeridad;

$\triangleright$ clasifique los costos y gastos por prioridades, tome decisiones para reducirlos o eliminarlos dependiendo de su importancia. 
Además, proponga una opción como el arrendamiento de equipos (Leasing Operativo) o la venta de equipos y arrendamiento simultáneo (Lease Back) para reducir costos y obtener liquidez;

$\triangleright$ implemente la técnica de negociación Gana-Gana (Win to Win);

$\triangleright$ se acerque a los bancos, los proveedores, los acreedores y el personal de la empresa para hacer acuerdos de pago. Asimismo, determine nuevos indicadores de capital de trabajo y evalúe la venta de cartera (Factoring) como opción para obtener liquidez;

$\triangleright$ cuando haya obligaciones financieras en moneda extranjera, analice la posibilidad de monetizarlas, mirando el costo financiero y la TRM;

$\triangleright$ fije nuevos indicadores de capital de trabajo, con base en los acuerdos;

$\triangleright$ fije nuevas políticas de venta con el director de mercadeo. Un nuevo enfoque al cliente ayuda a incrementar los ingresos. Esto significa: (1) retener los clientes actuales, corrigiendo los problemas existentes, adaptando el servicio a las barreras que puede presentar la crisis; (2) buscar nuevos clientes, aumentando la base de datos, que le ayuden a incrementar los ingresos; $y$, por último, (3) identificar nuevas formas efectivas de comunicar propuestas de valor al mercado;

$\triangleright$ tenga en cuenta los alivios ofrecidos por el Gobierno en materia de pago de impuestos, subsidios, otorgamiento de créditos y préstamos nuevos, tasas de interés, períodos de gracia, analizando la posibilidad de cumplimiento de pago futuro; sin descartar la posibilidad de incumplimiento;

$\triangleright$ analice la estructura administrativa para reducir costos. Para lograr esto, el gerente, primero, analiza la productividad del 
personal actual; después, hace una selección del personal de cara al futuro de la empresa, reteniendo el mejor talento para la empresa según sus fortalezas y capacidades. Sin embargo, si no es posible reubicar personal con rendimiento bajo, una opción es cambiar el contrato y reducir gastos, es decir, negociar. Finalmente, si la empresa lo permite, un cambio en la estructura organizacional hacia una estructura flexible facilita la modificación del esquema de costos;

$\triangleright$ analice la reducción de costos fijos proyectando el crecimiento de la empresa a corto y mediano plazo;

$\triangleright$ negocie con los empleados el pago de sus salarios;

$\triangleright$ haga acuerdos con los bancos, con los acreedores y todo tipo de obligaciones que haya incumplido o pueda incumplir, a raíz de la actuar situación económica y financiera;

$\triangleright$ si la posición de caja lo amerita, consiga nuevo capital por medio de los bancos o los inversionistas; sin descartar la capitalización: ¿hay suficientes garantías o respaldo para generar confianza con el cumplimiento de las actuales y nuevas obligaciones financieras?;

$\triangleright$ a partir de decisiones tomadas sobre los costos fijos y costos variables para adaptarse al cambio, determine la nueva participación de los costos fijos y los costos variables actuales;

$\triangleright$ finalmente, trabaje con el punto de equilibrio en diferentes modelos para obtener utilidades como se sugirió anteriormente.

- Una evaluación de los actuales sistemas de información: las metodologías de desarrollo o la infraestructura tecnológica óptima con el objetivo de llegar más rápido al cliente, para ofrecerle solución a sus problemas y generarle valor. El gerente tiene que pensar en mantener 
los ingresos que tenía antes de la crisis, esto es una inversión a largo plazo, las malas épocas ya pasaron.

- Un instrumento de control y orientación de la empresa desde la óptica financiera: el cuadro de indicadores de gestión es un instrumento que muestra diariamente la situación económica y financiera de la empresa (Anexo 5) para la toma de decisiones oportunas.

- Reservas de las utilidades obtenidas periódicamente para futuras eventualidades o contingencias: las utilidades totales no deben ser repartidas, en épocas de crisis la empresa puede insolventarse y desaparecer. Las empresas con reservas pueden subsistir más fácilmente en épocas de crisis e incertidumbre.

\section{Cómo implementar el proceso de innovación desde las finanzas}

En épocas de crisis e incertidumbre, el entorno es complejo por la alta volatilidad y dificultad para visualizar el futuro. Hay que adaptar nuevas estrategias buscando nuevas alternativas. La innovación trae sostenibilidad a los sistemas que rodean a la empresa, siempre y cuando se adopte como un proceso de generación de valor para los stakeholders o grupos de interés asociados a la empresa.

El gerente de finanzas está obligado a implementar la innovación no solo como un proceso para la creación de productos en el área de mercadeo, también para procesos de producción y administrativos, modelos de gestión, servicios y solución de necesidades al consumidor; ganar tiempo reduce costos e incrementa los ingresos cuando se concreta algo novedoso pero valioso para alguien.

Entonces, la innovación involucra algunos elementos fundamentales para que el proceso tenga resultados favorables. Para iniciar, hay que identificar y entender el problema, hay que innovar para desarrollar nuevas 
maneras de atender las necesidades de los clientes y de las personas, a nivel interno y externo. Construir las soluciones óptimas después de un análisis objetivo trabajando en equipo con los consumidores, los proveedores, los accionistas. El diseño de un modelo ágil lleva a obtener el cuadro de control financiero o indicadores de gestión financiera.

\section{Conclusiones}

No existen buenos ni malos negocios, en el mundo empresarial se necesitan líderes, empresarios innovadores que acepten el cambio y sean creativos y proactivos. La situación económica actual induce a una innovación disruptiva basada en una mentalidad flexible; cuando se presentan los cambios, hay que reinventar, repensar las estructuras actuales para crear nuevos productos y hacer nuevos negocios que se conviertan en líderes en el mercado. Se requiere de la mejora en la actual tecnología apoyada en herramientas que identifiquen cambios radicales en la empresa, para satisfacer las necesidades del consumidor.

Cuando la empresa se enfrenta a cambios excepcionales, el gerente de finanzas tiene que tomar el liderazgo empresarial, basándose en el análisis objetivo de los choques económicos que impactan la liquidez y el proceso productivo, analizando estrategias innovadoras para adaptar los procesos y los sistemas al cambio mediante la aplicación de herramientas que le permitan alcanzar los objetivos económicos y financieros fijados en la planeación de la empresa. A la vez, no puede perder de vista que estas herramientas deben estar enfocadas a la liquidez de la unidad empresarial, para fortalecer el flujo de tesorería que permita cumplir con sus obligaciones ante terceros, evitar costos inusuales y ofrecer la rentabilidad esperada sobre la inversión a los inversionistas.

Los economistas que han investigado y creado nuevo conocimiento con relación a la administración financiera empresarial coinciden en que las finanzas tienen un enfoque administrativo. Un ejemplo de esto es Ezra Solomon, quien señaló la relación del uso de los modelos de estrategia 
empresarial para optimizar los recursos económicos y financieros, el uso de herramientas de tecnología, software e inteligencia digital que se vienen implementando desde finales del siglo pasado y la participación en las áreas funcionales cuando se vean en peligro los ingresos.

En tiempos de crisis, una vez finaliza la auditoría inicial, la gerencia general, junto a la de finanzas, deben replantear la gerencia estratégica y redireccionar los planes hacia los objetivos propuestos, además de identificar las oportunidades, las fortalezas, las amenazas y debilidades de la empresa. Esto posibilita que la gestión financiera, la gestión de producción y mercadeo y la gestión de talento humano se desempeñen con una nueva visión hacia el futuro. Globalmente, el comportamiento de la economía ha cambiado el mundo de los negocios; los empresarios tienen que aceptar que los ciclos económicos siempre estarán presentes en el transcurso del tiempo y, en consecuencia, deben estar preparados para aceptar y afrontar el cambio. Toda situación de crisis es benéfica, trae oportunidades de cambio, innovación y crecimiento.

Ahora bien, para el gerente de finanzas, la innovación en épocas de incertidumbre significa gestionar proyectos innovadores en el diseño de un plan de negocios para los productos, los servicios y los procesos. Asimismo, su gestión está determinada por la aplicación de diferentes técnicas para desarrollar la innovación; por ejemplo, la identificación de cualidades, prácticas y enfoques en la empresa a partir del trabajo con el capital humano.

Ante la presentación de la incertidumbre, originada por una elevada expansión, la desaceleración económica o una depresión, hay que realizar un diagnóstico de la situación actual antes de actuar, que revele cómo las variables externas han impactado la empresa. El análisis del entorno permite ver cómo dicha situación afecta la demanda y cómo afecta cada una de las dependencias y áreas funcionales.

En ese orden de ideas, es recomendable que la gerencia de finanzas implemente un modelo de gerencia estratégica financiera a partir de la planeación y un estado de resultados por contribución (explicado en 
Horngren, 1994, p. 141), basado en una clara estructura de costos de la empresa. Asimismo, se aconseja que la gerencia incremente el margen de contribución y las utilidades operacionales, apoyado en la palanca de mercadeo, implementando la palanca de producción para reducir los costos y utilizando la regla de economía de escala y optimización de procesos. Igualmente, para reducir costos en las diferentes áreas funcionales, se sugiere que dicha dependencia aplique el justo a tiempo (лтт) en la cadena de abastecimiento en compañía del área de producción, con el director de talento humano, identificando el mejor talento, reubicando personal en áreas débiles, analizando el cambio de estructura organizacional para reducir gastos, buscar el cambio en la forma de contratación, orientándose a la eficiencia y productividad.

Otra de las propuestas derivadas del presente análisis es que se apliquen las palancas de operación y la palanca financiera según las necesidades en la dirección financiera. Además, que se analice la inversión en estructura de tecnología para lograr rápidas soluciones al consumidor y reducir procesos; puesto que la actual situación económica induce a reorientar los canales de comunicación. Es fundamental el evaluar nuevas fuentes de financiación, aprovechando las medidas de rescate con la política monetaria de flexibilización.

Paralelamente, la gerencia de finanzas tiene la responsabilidad de aplicar el estado de resultados por contribución, reafirmando los siguientes puntos: (1) un aumento del margen de contribución al incrementar los ingresos y reducir los costos variables; (2) una reducción del índice de estructura bajando los costos fijos y, por consiguiente, aumentando la utilidad de operación; (3) la aplicación del punto de equilibrio para generar utilidades, implementando el submodelo de contribución marginal, en el cual se puede ver la contribución de cada producto a la utilidad de operación. La contribución de cada producto permite asesorar al área de mercados en la venta de los productos más rentables; este instrumento ayuda en las nuevas formas de negociación. Por último, (4) la reducción del costo de capital modificando la estructura de endeudamiento. 
Sumado a esto, otra misión de la gerencia de finanzas es la elaboración del nuevo flujo de caja, el cual orienta las decisiones sobre la política de capital de trabajo. La empresa se quiebra por falta de liquidez, por lo que la gerencia debe implementar este instrumento fundamentado en la austeridad en el gasto. Simultáneamente, dicha austeridad va acompañada de elementos como la negociación de las deudas de los clientes ayudándoles en su flujo de caja, esto le permite asegurar sus ingresos, generar confianza y fidelidad; la negociación con sus acreedores, el pago de sus obligaciones, de ser necesario la gerencia puede mostrar a las entidades financieras su intención de pago mediante un plan que contemple, inclusive, la posibilidad de cancelar con nuevos descuentos por pago anticipado. Finalmente, la negociación del pago de las obligaciones y la forma de contratación con los empleados es otra estrategia para mitigar la falta de liquidez de una empresa.

Es conveniente que el gerente de finanzas construya y analice el ciclo de caja para ver la rotación de caja y sus efectos con las nuevas políticas de administración de capital de trabajo; para ello, debe tener a mano un modelo de medición del LAR para detectar necesidades extremas y provisiones diarias. La planeación financiera permite hacer análisis de sensibilidad y medir el comportamiento futuro de la empresa. Estas son estrategias claves para el futuro, puesto que posibilitan la prevención de contratiempos y constituye reservas para contingencias.

Para concluir, de acuerdo con las necesidades y puntos de vista del gerente, este puede elaborar un cuadro de mando con los principales indicadores financieros y técnicos que le muestren permanentemente la situación económica y financiera de la empresa y el riesgo de liquidez para tomar decisiones oportunas corrigiendo y ajustando lo necesario. En este proceso, aquel tiene en cuenta el modelo Dupont, un indicador integrado que le permite optimizar recursos y mejorar la rentabilidad sobre la inversión productiva (ROI) y la rentabilidad sobre los activos totales (ROA). El gerente juega con esas herramientas, hace análisis de sensibilidad para ver el efecto de estos indicadores en la rentabilidad. Es importante recordar 
que el gerente de finanzas toma decisiones con un enfoque administrativo, no solo hacia la evaluación de proyectos de inversión..

\section{Referencias}

Block, S. y Hirt, G. (2001). Fundamentos de Gerencia Financiera (9. a ed.). Mc Graw Hill Interamericana.

David, F. (1991). La Gerencia Estratégica (5. a ed.). Legis Editores.

Ehrhardt, M. y Brigham, E. (2006). Finanzas Corporativas (2. ${ }^{a}$ ed.). Cengage Learning Editores.

Haro, A. d. (2004). Medición y Control de Riesgos Financieros (3a ed.). Editorial Limusa S.A.

Horngren C., Sundem G. (1994). Introducción a la Contabilidad Administrativa (9. ${ }^{a}$ ed.). Prentice Hall Inc. México.

Kaplan, R. y Norton, D. (2005). Mapas Estratégicos: convirtiendo los activos intangibles en resultados tangibles. Ediciones Deusto.

La Covid-19 (coronavirus) hunde a la economía mundial en la peor recesión desde la Segunda Guerra Mundial. (8 de junio de 2020). Banco Mundial.

Mintzberg, H. y Brian, J. (1997). El Proceso Estratégico: conceptos, contextos y casos (1. ${ }^{\mathrm{a}}$ ed.). Prentice Hall Hispanoamericana.

Parra, A. (2014). The valuation of a company requires a wide vision of business. Gestión y Desarrollo, 2, 7-13.

Perspectivas económicas mundiales, enero 2020: crecimiento lento y desafíos normativos. (8 de enero de 2020). Banco Mundial.

Saldivar, A. (1999). Planeación Financiera de la Empresa. Tercera edición. Editorial Trillas S.A.

Sallenave, J. P. (1985). Gerencia y Planeación Estratégica. Editorial Norma.

Solomon, E. (1972). Teoría de la Administración Financiera. Ediciones Macchi.

W. Chan Kim y Mauborgne, R. (2015). La Estrategia del Océano Azul. Harvard Business Review. http://libertelia.org/wp-content/uploads/2016/04/Estrategiadel-Oceano-Azul.pdf

Zvi Bodie., R. Merton. (1999). Finanzas. Prentice Hall Hispanoamericana. 


\section{Anexos}

Anexo 1. Estado de resultados por contribución

\begin{tabular}{|c|c|}
\hline General & Por producto \\
\hline \multirow[t]{4}{*}{ Ventas } & Ventas por producto \\
\hline & Producto A \\
\hline & Producto B \\
\hline & Producto $\mathrm{C}$ \\
\hline Costos variables & Costos variables \\
\hline Producción & Producto A \\
\hline Administración & Producto B \\
\hline Finanzas & Producto $\mathrm{C}$ \\
\hline \multirow[t]{4}{*}{ Margen de contribución } & Margen de contribución \\
\hline & Producto A \\
\hline & Producto B \\
\hline & Producto $\mathrm{C}$ \\
\hline \multirow[t]{4}{*}{ Costo fijo + Costo financiero } & Costo fijo + Costo financiero \\
\hline & Producto A \\
\hline & Producto B \\
\hline & Producto $\mathrm{C}$ \\
\hline \multirow[t]{4}{*}{ Utilidad operacional } & Utilidad operacional \\
\hline & Producto A \\
\hline & Producto B \\
\hline & Producto C \\
\hline
\end{tabular}

Fuente: elaboración propia. 

una propuesta financiera

\section{Anexo 2. Modelo de flujo de caja mensual}

\begin{tabular}{|c|c|c|c|c|c|c|}
\hline Concepto & $\begin{array}{c}\text { Periodo } \\
\text { I }\end{array}$ & $\begin{array}{c}\text { Periodo } \\
\text { II }\end{array}$ & $\begin{array}{l}\text { Periodo } \\
\text { III }\end{array}$ & $\begin{array}{l}\text { Periodo } \\
\text { IV }\end{array}$ & $\begin{array}{c}\text { Periodo } \\
\text { XII }\end{array}$ & Total \\
\hline \multicolumn{7}{|l|}{ Ingresos } \\
\hline \multicolumn{7}{|l|}{ Ventas } \\
\hline \multicolumn{7}{|l|}{ Colocaciones } \\
\hline \multicolumn{7}{|l|}{ Total Ingresos } \\
\hline \multicolumn{7}{|l|}{ Egresos } \\
\hline \multicolumn{7}{|l|}{ Proveedores } \\
\hline \multicolumn{7}{|l|}{ Personal } \\
\hline \multicolumn{7}{|l|}{ Administrativos } \\
\hline \multicolumn{7}{|l|}{$\begin{array}{l}\text { Obligaciones } \\
\text { financieras }\end{array}$} \\
\hline \multicolumn{7}{|l|}{ Impuestos } \\
\hline \multicolumn{7}{|l|}{ Inversiones } \\
\hline \multicolumn{7}{|l|}{ Total egresos } \\
\hline \multicolumn{7}{|l|}{ Caja mínima } \\
\hline \multicolumn{7}{|l|}{ Total egresos } \\
\hline \multicolumn{7}{|l|}{ Diferencia } \\
\hline \multicolumn{7}{|l|}{ Financiamiento } \\
\hline \multicolumn{7}{|l|}{ Préstamos } \\
\hline \multicolumn{7}{|l|}{ Amortizaciones } \\
\hline \multicolumn{7}{|l|}{ Intereses } \\
\hline Total & & & & & & \\
\hline
\end{tabular}

Fuente: elaboración propia. 


\section{Anexo 3. Punto de equilibrio}

Momento en el que el margen de contribución (diferencia entre las ventas y los costos variables) cubre los costos fijos de la empresa. Al considerar la estructura de costos variables y costos fijos se puede dinamizar el estado de resultados a partir del margen de contribución y se pueden lograr puntos de equilibrio diferentes que permiten más flexibilidad en al tomar decisiones.

Las fórmulas para hallar los diferentes puntos de equilibrio son:

1. Punto de equilibrio en pesos (Ventas)

2. Punto de equilibrio en cantidades (Q)

3. Punto de equilibrio incluyendo gasto financiero

4. Punto de equilibrio con utilidad

5. Punto de equilibrio con utilidad y reducción de un porcentaje del precio de venta

6. Punto de equilibrio con utilidad después de impuestos

7. Punto de equilibrio con utilidad como porcentaje de la venta

8. Punto de equilibrio en efectivo

9. Ilustración cálculo punto de equilibrio Mezcla de Productos 
A continuación, se detallan dichas fórmulas:

1. Punto de equilibrio $=\frac{\text { Costo fijo }}{\text { Tasa de contribución }}=\frac{\mathrm{CF}}{(1-\mathrm{Cv} / \mathrm{Vta})}$ marginal

2. Punto de equilibrio $=\frac{\text { Costo fijo }}{\text { Margen de contribución }}=\frac{\mathrm{CF}}{(\mathrm{PVta}-\mathrm{CV})}$

3. Punto de equilibrio $=\frac{\text { Costo fijo }+ \text { Gasto financiero }}{\text { Tasa de contribución marginal }}=\frac{\mathrm{CF}+\mathrm{GF}}{(1-\mathrm{Cv} / \mathrm{Vta})}$

4. Punto de equilibrio $=\frac{\mathrm{CF}+\mathrm{GF}+\text { Utilidad }}{\text { Tasa de contribución }}=\frac{\mathrm{CF}+\mathrm{GF}+\mathrm{U}}{(1-\mathrm{Cv} / \mathrm{Vta})}$ marginal

5. Punto de equilibrio $=\frac{\mathrm{CF}+\mathrm{GF}+\text { Utilidad }}{\frac{\mathrm{CV}(\text { Razón Actual })}{(1-\% \text { Reducción Pvta })}}=\frac{\mathrm{CF}+\mathrm{GF}+\mathrm{U}}{\mathrm{CV}(\text { Razón Actual })}$

6. Punto de equilibrio $=\frac{\mathrm{CF}+\mathrm{GF}+\mathrm{UT} /(1-\mathrm{Tx})}{\text { Tasa de contribución }}=\frac{\mathrm{CF}+\mathrm{GF}+\mathrm{UT} /(1-\mathrm{Tx})}{(1-\mathrm{Cv} / \mathrm{Vta})}$ marginal

7. Punto de equilibrio $=\frac{\mathrm{CF}+\mathrm{GF}+\text { Utilidad }}{(\mathrm{TCMg})-\% \text { Venta }}=\frac{\mathrm{CF}+\mathrm{GF}+\mathrm{UT}}{(1-\mathrm{CV} / \mathrm{Vta})-\% \text { Venta }}$

8. Punto de equilibrio $=\frac{\mathrm{CF}-(\mathrm{dep}+\text { amort })}{\text { Tasa de contribución }}=\frac{\mathrm{CF}-(\mathrm{dep}+\mathrm{amort})}{(1-\mathrm{Cv} / \mathrm{Vta})}$ marginal 
9. Punto de equilibrio Mezcla de productos (ver tabla 1)

Tabla 1. Punto de equilibrio Mezcla de productos

\begin{tabular}{|c|c|c|c|c|}
\hline \multirow{2}{*}{ Concepto } & Producto & Producto & Producto & \multirow{2}{*}{ Totales } \\
\hline & $\mathbf{A}$ & B & $\mathrm{C}$ & \\
\hline Unidades & (1) & & & $(A+B+C)$ \\
\hline Participación & $(2)$ & & & \\
\hline Costo variable unitario & (3) & & & \\
\hline \multicolumn{5}{|l|}{ Materiales directos } \\
\hline \multicolumn{5}{|l|}{ MOD } \\
\hline \multicolumn{5}{|l|}{ CIF } \\
\hline Costo fijo total & & (4) & & \\
\hline Precio de venta unitario & $\$$ & $\$$ & $\$$ & \\
\hline Margen contribución unitario 1 & (5) & & & $(A+B+C)$ \\
\hline $\begin{array}{l}\text { Margen de contribución } \\
\text { unitario } 2\end{array}$ & (6) & & & МСт (7) \\
\hline Punto de equilibrio general & & & & (8) \\
\hline $\begin{array}{l}\text { Punto de equilibrio por } \\
\text { productos }\end{array}$ & (9) & & & $(A+B+C)$ \\
\hline
\end{tabular}

Fuente: elaboración propia. 


\section{Procedimiento:}

1. Determine las unidades a producir por producto, de acuerdo con el presupuesto. Obtenga el total a producir.

2. Obtenga la participación de cada producto, con base en el total de la producción estimada.

3. Determine el costo variable unitario por producto (materia prima + MOD + CIF) con la información proveniente de la contabilidad de costos.

4. Tome el costo fijo total de la empresa.

5. Tome el precio de venta unitario y réstele el costo variable unitario para determinar el margen de contribución unitario antes del punto de equilibrio. Sume estas partidas y obtenga el margen de contribución total de la empresa.

6. Tome el margen de contribución unitario calculado en 5 y multiplíquelo por la participación de cada producto frente a la producción total hallada en 2. Este es el margen de contribución por producto frente a la producción.

7. Sume el margen de contribución unitario 2 para obtener el margen de contribución general de la empresa.

8. Tome el costo fijo total y divídalo por el margen de contribución total. Este es el punto de equilibrio general en cantidades.

9. Aplique el porcentaje de la producción de cada producto hallado en 2 al punto de equilibrio general en cantidades hallado en 8 . Se encuentra el punto de equilibrio por producto. El valor de la sumatoria debe coincidir con el numeral 8. Elabore el estado de resultados con base en el punto de equilibrio por producto para conocer el mínimo a producir por producto y no tener pérdidas operacionales. 


\section{Anexo 4. Índice Dupont}

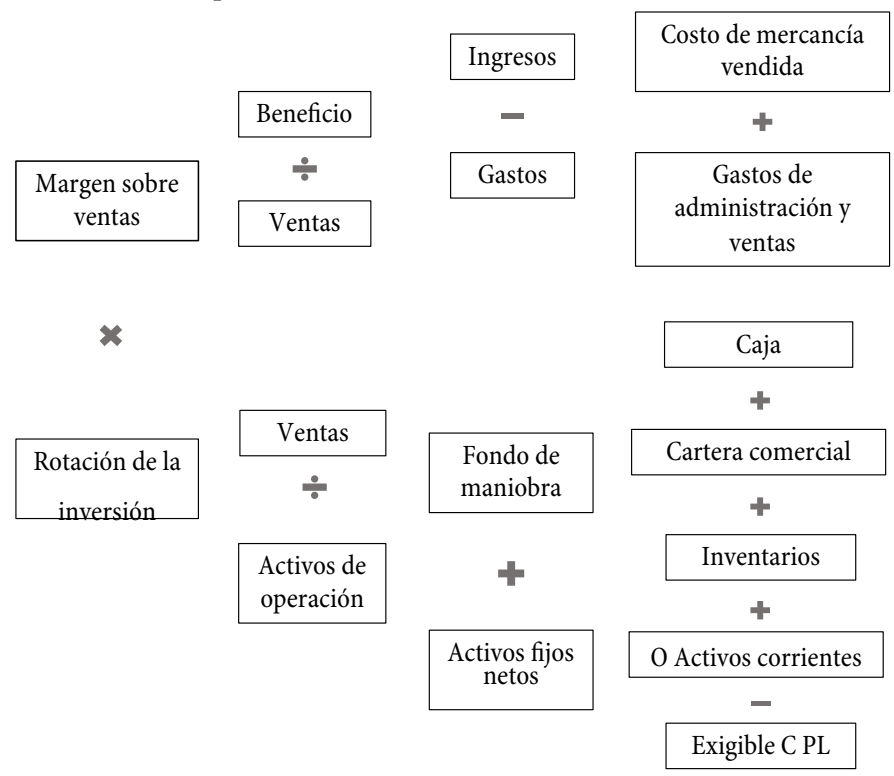

Fuente: elaboración propia.

El índice Dupont es el desarrollo de la rentabilidad de la inversión productiva (ROI) o el desarrollo de la rentabilidad sobre los activos totales (ROA). Es importante diferenciar la rentabilidad de estos dos conceptos: para el RoI se toma en el denominador la inversión operativa o aquellos activos que contribuyen directamente a la generación operativa de la empresa; valor diferente a los activos totales en el balance que se considera como ROA.

Para optimizar el resultado basta con tomar decisiones de política en la administración del capital de trabajo, reducción de gastos y costos, incremento de las ventas, eliminación de activos improductivos.

El margen de ventas es el resultado de dividir la utilidad neta entre las ventas. La rotación de los activos se determina al dividir las ventas netas entre el activo total. La utilidad neta proviene de tomar los ingresos, descontarle los egresos y deducir los impuestos. 
Los egresos están conformados por los costos de producción, los gastos de distribución y los gastos de administración.

Las ventas son el resultado de multiplicar el precio de venta por el volumen de las unidades vendidas, deducidas las devoluciones en ventas.

El activo total es la suma del activo fijo neto más los activos corrientes más otros activos. Los activos corrientes están conformados por el efectivo, los inventarios, las cuentas por cobrar comerciales, los deudores y los títulos valores. En algunas empresas existen otros conceptos que se clasifican como activos corrientes. Los activos fijos son aquellos considerados como la inversión bruta de capital que contribuye a la operación. Otros activos comprenden los conceptos que no se incluyen en los activos corrientes y no corrientes o fijos.

Esta composición del índice Dupont no es más que una herramienta que permite a la gerencia financiera mejorar la rentabilidad a partir de la eficiencia con la que se manejen los activos productivos de la empresa. La optimización en la rentabilidad económica depende de la mejora en el margen de ventas y la mejora en la tasa de rotación del activo; en consecuencia, el roA y ROI optimizados depende de las decisiones que se tomen desde la gerencia financiera, sobre las cuentas que componen el margen de ventas y la rotación del activo total y así sucesivamente los indicadores que las componen. 
Anexo 5. Cuadro de mando y control financiero

\begin{tabular}{|c|c|c|}
\hline Liquidez & Productividad & Rentabilidad \\
\hline $\mathrm{CxC}$ & Producción/HH & ROI \\
\hline $\mathrm{CxP}$ & $\begin{array}{l}\text { Producción/ } \\
\text { Trabajadores }\end{array}$ & ROA \\
\hline Inventarios & $\begin{array}{c}\text { Remuneración/ } \\
\text { Productividad } \\
\text { Laboral }\end{array}$ & ROE \\
\hline KT & & EVA \\
\hline Ingresos & & Ebitda \\
\hline Egresos & & Margen Ebitda \\
\hline Saldo Caja & & RION \\
\hline \multicolumn{3}{|l|}{ LAR } \\
\hline Utilidades & Endeudamiento & Eficiencia \\
\hline $\begin{array}{c}\text { Utilidad } \\
\text { Operacional }\end{array}$ & Corto Plazo & $\begin{array}{c}\text { Ventas/ Total } \\
\text { Empleados }\end{array}$ \\
\hline Utilidad Neta & Largo Plazo & $\begin{array}{c}\text { Costos Servicios/ } \\
\text { Costos } \\
\text { Producción }\end{array}$ \\
\hline \multirow[t]{2}{*}{ Margen neto } & $\begin{array}{l}\text { Abono servicio a } \\
\text { la deuda }\end{array}$ & $\begin{array}{c}\text { Ventas/ Activos } \\
\text { Productivos }\end{array}$ \\
\hline & $\begin{array}{l}\text { Concentración } \\
\text { corto plazo - } \\
\text { largo plazo }\end{array}$ & \\
\hline
\end{tabular}

Fuente: elaboración propia. 


\section{Anexo 6. Cálculo del EVA}

\begin{tabular}{|l|c|l|l|l|l|}
\cline { 2 - 6 } \multicolumn{1}{l|}{} & Año 1 & Año 2 & Año 3 & Año 4 & Año 5 \\
\hline Utilidad Operacional & $\$$ & & & & \\
\hline Impuestos & $\$$ & & & & \\
\hline OCDI - NOPAT & $\$$ & & & & \\
\hline ROIC o RION & $\%$ & & & & \\
\hline Costo Financiero & $\$$ & & & & \\
\hline EVA & $\$$ & & & & \\
\hline EVA & $\%$ & & & & \\
\hline Valor de mercado & $\%$ & & & & \\
\hline Tasa de Impuestos & $\$$ & & & & \\
\hline Costo de Capital & & & & & \\
\hline Activos Operativos & & & & \\
\hline
\end{tabular}

Fuente: elaboración propia.

\section{Anexo 7. Indicador EVA}

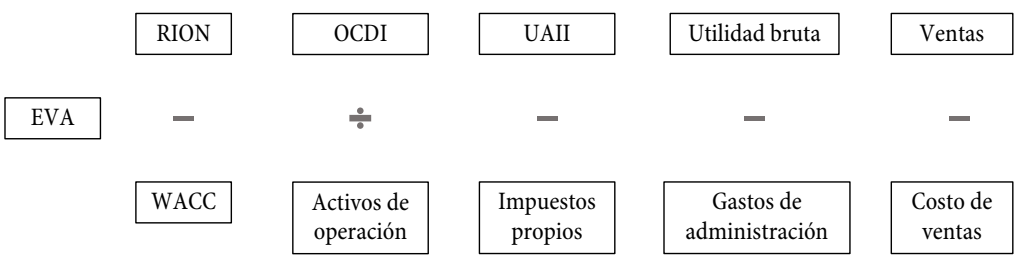

Fuente: elaboración propia. 
Para optimizar el Valor Económico Agregado hay que tomar decisiones en relación con los siguientes conceptos:

- Eliminar activos fijos no productivos.

- Reducir los gastos operacionales innecesarios.

- Incrementar los ingresos.

- Invertir en proyectos que generen van positivo. Mejorar el costo financiero, negociando tasas bajas en las entidades financieras. $\mathrm{Si}$ tiene deudas con entidades bancarias en el exterior; analice

el costo financiero en Colombia frente al costo financiero externo, la devaluación impacta el costo financiero, cambie la estructura de endeudamiento si es más costosa la deuda en el exterior.

Por lo anterior, si se incrementa la utilidad de operación (UAII) se mejora el valor económico agregado EVA. Le aplicamos la tasa de impuestos específica de la empresa, este valor lo relacionamos con la inversión en activos productivos, tomados del balance general, y el rendimiento de estos activos se incrementa. Si el costo de capital (CCPP) se reduce hay una mayor generación de EvA. Se incrementa el valor de la empresa. 